\title{
Usos del suelo en las cuencas hidrográficas de los humedales del Alto Guadalquivir: Importancia de una adecuada gestión
}

\author{
Fernando Ortega, Gema Parra y Francisco Guerrero*
}

Departamento de Biología Animal, Biología Vegetal y Ecología. Facultad de Ciencias Experimentales. Universidad de Jaén. Campus de Las Lagunillas s/n. E-23071 Jaén.

* Corresponding author: fguerre@ujaen.es

\section{RESUMEN}

Usos del suelo en las cuencas hidrográficas de los humedales del Alto Guadalquivir: Importancia de una adecuada gestión

Se ha realizado la delimitación y valoración de los usos del suelo en la cuenca hidrográfica de 22 humedales de la comarca del Alto Guadalquivir. Para dicha valoración se ha diferenciado entre cultivos (herbáceos y arbóreos); vegetación natural (monte bajo), terrenos forestales, pastizales, humedales desecados y terrenos urbanizados. El olivar es la actividad que mayor ocupación de suelo presenta en el territorio estudiado. Las actividades regularmente desarrolladas en el cultivo intensivo del olivar provocan el incremento de perturbaciones que repercuten sobre toda la cuenca de drenaje y particularmente en la calidad de las aguas y la conservación del humedal. Los resultados obtenidos se discuten en el contexto de la conservación de los humedales de la comarca del Alto Guadalquivir.

Palabras clave: Humedales, Paisajes mediterráneos, Cuencas de drenaje, Usos del suelo, Olivar.

\begin{abstract}
Land uses in the hydrographic basins of the Alto Guadalquivir wetlands: The importance of a suitable management

Drainage basin demarcation and the land uses identification have been carried out in 22 wetlands from the Alto Guadalquivir area. The land uses identified were crops (herbaceous and tree crops); natural vegetation; forests; grazing land; desiccated wetland and urban areas. Olive tree growing was the predominant soil use detected. The regular activities carried out in the olive intensive agriculture generate increased perturbations over the whole drainage basin and especially on the water quality and wetland conservation. The results obtained are discussed within the context of the Alto Guadalquivir wetland conservation.
\end{abstract}

Keywords: Wetlands, Mediterranean landscapes, Catchment areas, Land uses, Olive tree crops.

\section{INTRODUCCIÓN}

Los humedales se encuentran entre los espacios naturales con mayor valor ambiental, tanto por el mantenimiento de una elevada diversidad biológica, lo que establece claramente su relevancia ecológica; como por la importancia que han tenido para el desarrollo de la humanidad, desde una perspectiva cultural e histórica, económica, como fuente de riqueza sostenible, y científica (Bernáldez, 1987; Williams, 1999).
El estudio de los humedales ha permitido en los últimos años poner de manifiesto la relevancia que las cuencas hidrográficas tienen sobre el funcionamiento de estos ecosistemas acuáticos (véase por ejemplo Gibson et al., 1995; Ulrich, 1997; Morales-Baquero et al., 1999; Morales-Baquero \& Conde-Porcuna, 2000; Mäler, 2000; Matias, 2001; Istvánovics \& Somlyódy, 2001), siendo por ello actualmente consideradas como los instrumentos más adecuados en la gestión de los humedales 
y en la conservación de su integridad ecológica original (INIMA, 1996; Ors, 1999).

Una buena gestión de las cuencas de drenaje es, por tanto, fundamental para mantener la calidad ecológica de estos ecosistemas, ya que los humedales dependen directamente de los procesos hidrogeomorfológicos, biológicos y humanos (agricultura, deforestación, regulación, urbanización, etcétera) que se producen en los ecosistemas terrestres que drenan hacia sus cubetas. Por todo ello, resultaría imposible comprender completamente el estado actual de un humedal sin tener presentes los usos del suelo y los cambios producidos en su cuenca hidrográfica.

Esta valoración sobre la importancia de una correcta gestión de las cuencas de drenaje en la conservación de los humedales ha quedado recogida en diversos planes y convenios, tanto en el ámbito internacional (convenio de Ramsar y convenio sobre la Biodiversidad Biológica), como nacional (Plan Estratégico Español para la Conservación y el Uso Racional de los Humedales) y regional (Plan Andaluz de Humedales).

Andalucía posee un conjunto de humedales muy destacable a nivel de la Península Ibérica, con la presencia de una gran variedad de tipos ecológicos, algunos de los cuales son únicos en el contexto de la Unión Europea (Molina et al., 2002). Dentro de esta comunidad autónoma, la comarca del Alto Guadalquivir presenta uno de los conjuntos palustres menos conocidos y estudiados, con una amplia variedad de tamaños y tipologías presentes (Ortega et al., 2003).

Este desconocimiento ha llevado a que sólo tres de ellos hayan sido incluidos hasta el momento dentro de la red de espacios protegidos de Andalucía. Sin embargo, estudios recientes (Ortega et al., 2004) han puesto de manifiesto la existencia de un conjunto de humedales, agrupados bajo la denominación de red palustre "lagunas del Alto Guadalquivir", que por sus valores naturales están en vías de ser incluidos dentro el Inventario Andaluz de Humedales y en las propuestas andaluzas para formar parte de la Red Natura 2000.

De este modo, este estudio se plantea como objetivo primordial la delimitación y la descripción de los usos del suelo en las cuencas de drenaje de los humedales incluidos en la red palus- tre "lagunas del Alto Guadalquivir". Este objetivo es fundamental para el conocimiento y funcionamiento de estos humedales, proporcionando de este modo una herramienta básica para la correcta gestión y conservación de las zonas húmedas. Los resultados de este estudio podrán servir de base comparativa para evaluar futuros procesos de cambios en los usos del suelo y su consiguiente repercusión sobre los humedales del Alto Guadalquivir.

\section{ÁREA DE ESTUDIO}

La comarca del Alto Guadalquivir se localiza al Nordeste de Andalucía (España), englobando la casi totalidad de la provincia de Jaén y la parte más oriental de la provincia de Córdoba.

Uno de los elementos característicos de esta comarca es la presencia del valle del río Guadalquivir y la gran actividad de tipo agrícola desarrollada en el mismo, hecho que ha conducido a la degeneración de sus ecosistemas naturales en general y de los humedales en particular (Guerrero et al., 2002 y 2005).

La mayoría de éstos son lagunas estacionales de variado tamaño y origen geológico, habiendo sido inventariadas hasta la actualidad un total de 90 humedales (Ortega et al., 2003). De este colectivo se ha seleccionado para este estudio 22 humedales (Fig. 1; Tabla 1) englobados bajo la red palustre "lagunas del Alto Guadalquivir'" (Ortega et al., 2004) en función de su estado de conservación y de la valoración de sus comunidades acuáticas.

\section{MATERIAL Y MÉTODOS}

Para la descripción y estudio pormenorizado de los usos del suelo llevada a cabo en las cuencas de drenaje de los humedales incluidos en este estudio, se han utilizado ortofotografías digitales a color a escala 1:10.000 (Junta de Andalucía, 2003); cartografía a escalas 1:50.000 y 1:10.000, para la correcta delimitación de las cuencas vertientes, así como diversas jornadas de campo necesarias para cumplimentar a detalle los aspectos que no han podido ser realizados en gabinete. 


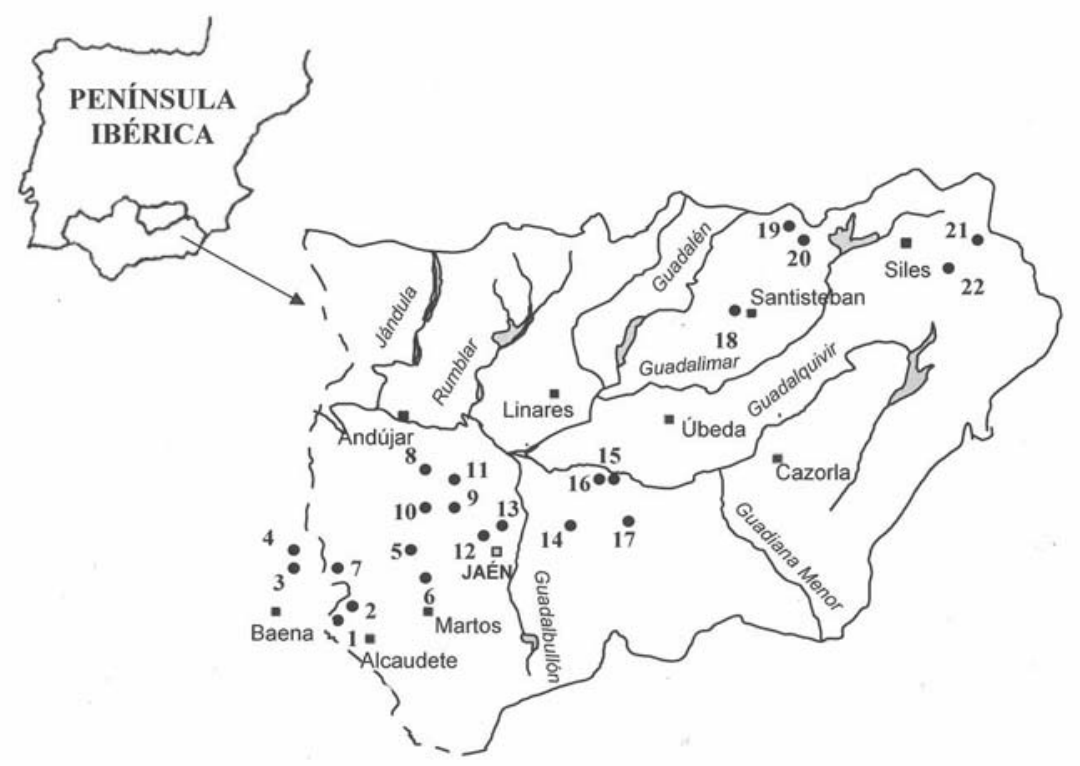

Figura 1. Comarca del Alto Guadalquivir y localización de los humedales incluidos en este estudio. 1: Laguna Honda; 2: Laguna del Chinche; 3: Laguna de la Quinta; 4: Laguna del Rincón del Muerto; 5: Laguna de Hituelo; 6: Laguna de Naranjeros; 7: Laguna de las Ceras; 8: Laguna del Ranal; 9: Laguna de Casillas; 10: Laguna de las Navas; 11: Laguna de Villardompardo; 12: Laguna de Garcíez; 13: Laguna de Prados del Moral; 14: Laguna de Brujuelo; 15: Laguna Grande; 16: Laguna Chica; 17: Laguna de Argamasilla; 18: Laguna de Santisteban; 19: Laguna de Perales; 20: Laguna de Pedernoso; 21: Laguna de Siles; 22: Laguna de Orcera. Alto Guadalquivir region and location of the wetlands included in this study. 1: Laguna Honda; 2: Laguna del Chinche; 3: Laguna de la Quinta; 4: Laguna del Rincón del Muerto; 5: Laguna de Hituelo; 6: Laguna de Naranjeros; 7: Laguna de las Ceras; 8: Laguna del Ranal; 9: Laguna de Casillas; 10: Laguna de las Navas; 11: Laguna de Villardompardo; 12: Laguna de Garcíez; 13: Laguna de Prados del Moral; 14: Laguna de Brujuelo; 15: Laguna Grande; 16: Laguna Chica; 17: Laguna de Argamasilla; 18: Laguna de Santisteban; 19: Laguna de Perales; 20: Laguna de Pedernoso; 21: Laguna de Siles; 22: Laguna de Orcera.

Con todo ello se ha procedido a una delimitación de la cuenca hidrográfica superficial de cada humedal, tras lo cual se ha acometido la identificación y cuantificación en términos de superficie ocupada (programas Mulhacen y ArcView), de cada uno de los usos del suelo.

Se ha diferenciado entre cultivos (herbáceos y arbóreos); vegetación natural (monte bajo), terrenos forestales, pastizales, humedales desecados y terrenos urbanizados (normalmente como cortijos aislados). Dentro de los cultivos se ha diferenciado entre la presencia de cultivos de regadío o de secano. Igualmente, dentro de los cultivos arbóreos (entre 100-125 árboles por hectárea), se ha hecho hincapié en la distinción de la edad de las plantaciones, considerando olivares maduros a aquellos que presentan una edad superior a 10 años. Finalmente destacar la localización y cartografiado de antiguas zonas húmedas, incluidas en las cuencas de algunos de los humedales ob- jeto de estudio, que actualmente se encuentran desecadas por acción antrópica, y que constituyen áreas de gran importancia que pueden suponer zonas potenciales para la aplicación de un futuro plan de restauración ecológica.

\section{RESULTADOS}

En la Tabla 1 se presentan algunas características limnológicas de los humedales estudiados. Como puede observarse, estos humedales se sitúan entre los 265 y los 1280 metros de altitud, habiendo sido clasificados en dos tipologías (Ortega et al., 2003), humedales asociados a zonas de montaña (lagunas de Siles y Orcera) y asociados al valle del río Guadalquivir (el resto). La mayor parte de ellos presentan, por su ubicación geográfica, un régimen hídrico (según Cirujano, 1995), que las sitúa como lagunas o humedales estacionales 
anuales (de inundación cada año) o estacionales habituales (con inundación cada 3-5 años).

En la Tabla 2 se exponen para cada humedal estudiado: (i) el área de su cuenca de drenaje superficial, y (ii) la superficie en hectáreas que ocupan los distintos usos del suelo presentes en cada cuenca.

Los resultados obtenidos muestran que el cultivo del olivar es el uso del suelo que presenta mayor representación dentro de las cuencas, ocupando el $69 \%$ del total del territorio estudiado y siendo por ello el principal uso del territorio en 18 de los 22 humedales de la comarca. Sin embargo, si de este cálculo eliminamos los dos humedales de montaña, este valor aumenta hasta el $90 \%$ de ocupación. Otro factor a considerar es la palpable transformación que viene produciéndose en los últimos diez años en la comarca, observándose una tendencia de cambio que ha provocado que el olivar tradicional de secano esté en retroceso frente al más productivo olivar intensivo de regadío, que ya constituye el uso predo- minante en la cuenca de 7 de los 18 humedales anteriormente mencionados.

En relación con la edad de las plantaciones de olivar, la mayor parte de las hectáreas de olivar presentes en las cuencas hidrográficas de los humedales del Alto Guadalquivir se corresponden con un olivar maduro, presentándose los olivares jóvenes en aquellas cuencas con cultivos tradicionales de herbáceas de secano que se están reconvirtiendo en olivar, por las exigencias del mercado y la política agraria predominante.

Los cultivos de cereal y girasol de secano son un tipo de uso en proceso de desaparición en el Alto Guadalquivir. En términos de ocupación suponen únicamente el $5 \%$ del total del territorio estudiado, apareciendo como cultivo predominante en dos humedales, la laguna de Santisteban y la laguna de Perales, ambos localizados en la sub-comarca del Condado, al norte de la provincia de Jaén. Esta sub-comarca se caracteriza por el mantenimiento de los usos tradicionales y por una tardía expansión del olivar. En el resto

Tabla 1. Características limnológicas de los humedales estudiados. Los epígrafes se corresponde con Ah: área del humedal (hectáreas); Alt: altitud (metros); P: profundidad máxima detectada (metros); Rh: régimen hídrico (P: permanente; Ea: Estacional anual; Eh: Estacional habitual); Cond: rango de conductividad observado $\left(\mathrm{mS} \mathrm{cm}^{-1}\right)$. Limnological features of the studied wetlands. Ah: wetland area (hectares); Alt: altitude (meters); P: maximum detected depth (meters); Rh: hydrologic regime (P: permanent; Ea: annual seasonal; Eh: habitual seasonal); Cond: range of measured conductivity $\left(\mathrm{mS} \mathrm{cm}^{-1}\right)$.

\begin{tabular}{lcccccc}
\hline Humedal & Coordenadas UTM & Ah & Alt & P & Rh & Cond \\
\hline Honda & 30SUG9961 & 9.9 & 446 & 3.16 & P-Ea & $12.1-198.2$ \\
Chinche & 30SUG9863 & 4.7 & 452 & 1.07 & Eh & $2.3-8.1$ \\
Quinta & 30SUG8679 & 7.7 & 289 & 3.15 & Eh & $0.8-33.9$ \\
Rincón Muerto & 30SUG8779 & 4.2 & 265 & 1.66 & Ea & $12.3-187.6$ \\
Hituelo & 30SVG0679 & 3.8 & 476 & 2.64 & Ea & $0.6-1.1$ \\
Naranjeros & 30SVG0978 & 5.2 & 508 & 4.56 & $\mathrm{P}$ & $0.5-0.9$ \\
Las Ceras & 30SVG0175 & 1.8 & 396 & 1.40 & $\mathrm{Eh}$ & $0.3-0.6$ \\
El Ranal & 30SVG0697 & 10.7 & 340 & 0.81 & $\mathrm{Ea}$ & $0.8-2.1$ \\
Casillas & 30SVG1083 & 2.7 & 442 & 2.58 & $\mathrm{Ea}$ & $0.2-0.6$ \\
Las Navas & 30SVG0486 & 3.5 & 378 & 2.23 & $\mathrm{Ea}$ & $0.4-0.7$ \\
Villadonpardo & 30SVG1489 & 1.7 & 360 & 3.18 & $\mathrm{Ea}$ & $0.8-2.6$ \\
Garcíez & 30SVG2389 & 7.9 & 441 & 3.55 & $\mathrm{Eh}$ & $0.5-3.8$ \\
Prados Moral & 30SVG2989 & 3.8 & 389 & 1.20 & $\mathrm{Eh}$ & $0.6-7.4$ \\
Brujuelo & 30SVG4191 & 4.2 & 458 & 2.12 & $\mathrm{Ea}$ & $12.1-68.2$ \\
Grande & 30SVG5098 & 22.9 & 368 & 3.50 & $\mathrm{P} / \mathrm{Ea}$ & $0.7-1.3$ \\
Chica & 30SVG5098 & 5.8 & 370 & 0.80 & $\mathrm{Eh}$ & $0.6-2.0$ \\
Argamasilla & 30SVG5392 & 4.8 & 484 & 2.20 & $\mathrm{Ea}$ & $0.8-2.4$ \\
Santisteban & 30SVH8134 & 3.0 & 637 & 0.90 & $\mathrm{Eh}$ & $0.5-1.4$ \\
Perales & 30SVH9548 & 5.2 & 757 & 1.05 & $\mathrm{Eh}$ & $0.07-0.1$ \\
Pedernoso & 30SWH0047 & 1.4 & 724 & 1.10 & $\mathrm{Eh}$ & $0.1-0.2$ \\
Siles & 30SWH4249 & 1.3 & 1280 & 2.34 & $\mathrm{P} / \mathrm{Ea}$ & $0.4-0.6$ \\
Orcera & 30SWH3542 & 0.5 & 1270 & 1.73 & $\mathrm{Ea}$ & $0.8-2.3$ \\
\hline
\end{tabular}


Tabla 2. Resultados obtenidos de la delimitación de las cuencas y descripción de los usos del suelo en los humedales de la red palustre del Alto Guadalquivir. Los epígrafes se corresponden con AC: área de la cuenca de drenaje del humedal; Omr: olivar maduro de regadío; Ojr: olivar joven de regadío; Oms: olivar maduro de secano; Ojs: olivar joven de secano; Chs: cultivos herbáceos de secano; P: pastizal; Ld: Laguna desecada; Mb: matorral o monte bajo; Cf: cultivos forestales y Ur: urbanizaciones o construcciones humanas. Todos los datos de superficie aparecen en hectáreas. Results of the drainage area delimitation and description of land uses in the Alto Guadalquivir wetlands. Epigraphs are as follows: AC: wetland catchment area; Omr: irrigated mature olive grove; Ojr: irrigated young olive grove; Oms: non-irrigated mature olive grove; Ojs: non-irrigated young olive grove: Chs: non-irrigated herbaceous crops; P: pasture; Ld: dry lagoon; Mb: brushes or scrubland; Cf: forest crops; and Ur: urban areas or human constructions. All data are presented in hectares.

\begin{tabular}{crrrrrrrrrrr}
\hline Humedal & AC & Omr & Ojr & Oms & Ojs & Chs & P & Ld & Mb & Cf & Ur \\
\hline Honda & 86.2 & 83.6 & - & - & - & - & - & 0.7 & 1.9 & - & - \\
Chinche & 82.6 & 80.7 & - & - & - & - & - & 1.9 & - & - & - \\
Quinta & 70.8 & - & - & 18.7 & 32.8 & 19.2 & - & - & 0.1 & - & - \\
Rincón Muerto & 43.9 & - & - & 29.5 & 9.7 & 1.2 & - & 3.3 & - & - & 0.2 \\
Hituelo & 29.0 & - & - & 28.6 & - & - & - & - & 0.4 & - & - \\
Naranjeros & 105.9 & 23.5 & - & 78.3 & - & - & - & 3.2 & 0.3 & - & 0.6 \\
Las Ceras & 14.8 & 12.4 & 1.2 & - & - & - & - & - & 0.1 & - & 1.1 \\
El Ranal & 195.1 & - & - & 142.4 & 40.5 & 12.2 & - & - & - & - & - \\
Casillas & 22.7 & - & - & 22.7 & - & - & - & - & - & - & - \\
Las Navas & 67.6 & - & - & 52.1 & - & 15.5 & - & - & - & - & - \\
Villadonpardo & 59.5 & - & - & 54.9 & - & - & - & - & 4.6 & - & - \\
Garciez & 103.3 & - & - & 96.2 & - & - & - & - & 1.7 & - & 5.4 \\
Prados Moral & 16.2 & - & - & 11.5 & 3.8 & - & - & - & 0.9 & - & - \\
Brujuelo & 156.3 & 96.0 & 49.1 & - & - & - & - & 3.3 & 7.5 & - & 0.4 \\
Grande + Chica & 121.9 & 121.9 & & - & - & - & - & - & - & - & - \\
Argamasilla & 43.3 & 41.4 & - & - & - & - & - & - & 1.9 & - & - \\
Santisteban & 25.8 & - & - & - & - & 17.7 & 6.4 & - & - & - & 1.7 \\
Perales & 14.2 & - & - & 5.4 & - & 7.7 & - & - & 1.1 & - & - \\
Pedernoso & 14.9 & - & - & 7.9 & - & 4.9 & - & - & 2.1 & - & - \\
Siles & 300.0 & - & - & - & - & - & 40.5 & - & - & 259.5 & - \\
Orcera & 84.9 & - & - & - & - & - & - & - & 7.7 & 77.2 & - \\
\hline
\end{tabular}

de las zonas agrícolas sólo aparecen rodales aislados que ocupan poca extensión en la cuenca y cuyo cambio a cultivo de olivar es inminente.

Los cambios en los usos del suelo anteriormente mencionados también han afectado de forma directa a la ganadería de la comarca, antaño ampliamente extendida, y que en la actualidad ha quedado relegada a las zonas de montaña. Este cambio ha originado igualmente una modificación en los usos del suelo, de modo que la utilización de praderas y pastizales de secano, así como el uso de una buena parte de estas lagunas y de su cuenca de drenaje como zona de pastoreo o como zonas de abrevadero (Madero et al., 2004), ha quedado en un segundo plano. En la actualidad sólo son aun utilizadas en este sentido las lagunas de Santisteban y Perales, nuevamente en la sub-comarca del Condado.

Las zonas de matorral y monte bajo presentes en la zona de estudio constituyen ves- tigios de la vegetación natural que en tiempos pasados cubría la comarca del Alto Guadalquivir y que, posteriormente, pasó a formar parte de padrones o linderos que servían para la separación y delimitación de las fincas. Los resultados obtenidos señalan que 13 de las lagunas estudiadas muestran restos de la antigua vegetación natural potencial de la zona, pero con un porcentaje de ocupación del territorio de tan sólo el $2 \%$. En la actualidad, la mayoría de estos padrones han desaparecido bajo la acción de herbicidas y pesticidas para facilitar las labores agrícolas, restando únicamente pequeños retazos en las partes más abruptas o rocosas de la cuenca de los humedales, en los que se refugian, en algunos casos, especies y comunidades catalogadas por la Directiva Hábitat de la UE.

La presencia de cultivos forestales se circunscribe únicamente a las cuencas de las lagunas de Orcera y Siles, localizadas en la sub-comarca de 
Tabla 3. Humedales desecados que se integran dentro de alguna de las cuencas de drenaje incluidas en el presente estudio. Dry wetlands located in some of the drainage areas included in the present study.

\begin{tabular}{ccccc}
\hline Humedal & Cuenca & Area (ha) & Estado actual & Uso actual \\
\hline Laguna de Casasola & Rincón Muerto & 2.5 & Colmatada, cultivada & Girasol \\
Laguna Honda & Honda & 1.1 & Drenada, colmatada & Pastizal \\
Laguna del Chinche II & Chinche & 2.1 & Drenada, colmatada & Pastizal \\
Laguna de Rumpisaco & Naranjeros & 1.6 & Drenada, colmatada & Pastizal \\
Laguna de Cirueña & Brujuelo & 2.8 & Drenada, colmatada & Carrizal \\
\hline
\end{tabular}

la Sierra de Segura, al límite con la provincia de Albacete. En ella se llevaron a cabo antiguas repoblaciones de pinares (Pinus nigra y Pinus pinaster) para la explotación de resina y madera de los montes de dominio publico. En ambos casos se trata de pinares maduros que ocupan la mayor parte del territorio junto a zonas de pastizal y retazos de encinar o matorral.

Incluidos en las cuencas de algunos de los humedales objeto de estudio, aparecen cubetas adyacentes de distinto tamaño, que se encuentran en la actualidad desecadas y colmatadas por la acción agrícola, así como drenadas hacia la cubeta principal (Tabla 3). Estos humedales se comportan en la actualidad como ocasionales, acumulando agua durante poco tiempo sólo en años lluviosos y drenando la sobrante al humedal principal.

Finalmente indicar que en la periferia de las lagunas aparecen diversos tipos de construcciones humanas, que van desde pueblos o aldeas como Santisteban del Puerto y Garcíez, a zonas de urbanizaciones recreativas, almazaras y cortijos, como sucede por ejemplo, en las cuencas de las lagunas de Naranjeros, Rincón del Muerto, Las Ceras y Brujuelo. La influencia de estas construcciones es diversa y en general constituyen un impacto negativo, ya sea por las actividades de desecación o modificación realizadas en la vecina laguna para su uso agrícola, ganadero, recreativo, etcétera. Igualmente, los recientes cambios agrícolas de la comarca han fomentado en los últimos años la transformación de las cubetas lacustres en balsas de riego para el olivar (Ortega et al., 2005), así como en balsas de alpechín de las almazaras, actividad esta última, que afecta a varios humedales en la comarca (lagunas de Garcíez y del Ranal).

\section{DISCUSIÓN}

La región mediterránea ha sufrido desde tiempos remotos fuertes transformaciones a consecuencia de la actividad humana, siendo las actividades pastoril y agrícola las que mayores repercusiones han tenido en los cambios del paisaje registrados en esta región. La comarca del Alto Guadalquivir muestra una problemática ambiental que se manifiesta claramente asociada a un proceso de intensificación agrícola (Guerrero et al., 2005). Los resultados obtenidos muestran la dominancia del olivar sobre el resto de cultivos y usos del suelo; quedando igualmente patente la progresiva transformación del olivar tradicional de secano en un olivar intensivo de regadío (Moraga, 2002), proceso que se está llevando a cabo no sólo en el Alto Guadalquivir, sino también en el resto de Andalucía.

El cultivo del olivo estructura la economía de la mayoría de los pueblos de esta comarca, actuando como catalizador cultural de la multitud de procesos económicos, sociales y culturales de esta sociedad (Anta, 2002; Palacios, 2002). Sin embargo, desde un punto de vista ambiental y de conservación de la naturaleza, la implantación de cualquier monocultivo o sistema de sobreexplotación y la profunda transformación sufrida por ello en el territorio, genera fuertes transformaciones tanto en los agrosistemas tradicionales como en los ecosistemas adyacentes (Boutin \& Jobin, 1998).

En este sentido hay que señalar que el olivar es uno de los cultivos en los que las pérdidas de suelo son más elevadas, muy superiores a las observadas en cultivos de cereal, girasol o en zonas de pastizal y matorral, con estimaciones en los olivares andaluces de una pérdida media de 80 
toneladas de suelo por hectárea y año, pérdidas que se incrementan en territorios con fuerte pendiente (Pastor et al., 1996). Si tenemos en cuenta que la tasa media de formación de suelo en condiciones favorables es de una tonelada de suelo por hectárea y año (Porta et al., 1994), podemos tener una clara constatación de uno de los principales problemas ambientales del territorio estudiado.

Del mismo modo, las actividades generadas en los agrosistemas se basan fundamentalmente en la consecución de resultados de cosecha, lo que lleva asociado un incremento de aportes en forma de energía auxiliar o productos agroquímicos. Este hecho se refleja de dos maneras bien diferentes en los ecosistemas acuáticos. En primer lugar a través de una excesiva utilización de los recursos hídricos, tanto superficiales como subterráneos, con el incremento de pozos de extracción, construcción de balsas de regadío, desecación de humedales, etcétera (Ortega et al., 2003; 2005), efectos que van asociados al incremento de cultivos en regadío y que va a tener especial re- percusión en los humedales y en su balance hídrico anual. En segundo lugar, estas actividades se manifiestan en los efectos de los productos agroquímicos sobre estos ecosistemas acuáticos.

Para reflejar este hecho, y aunque la estimación de las fuentes de nutrientes alóctonas a los sistemas acuáticos es de difícil obtención (véase por ejemplo Irvine et al., 2000), se ha realizado una valoración de los aportes de nitrógeno y fósforo total utilizando los coeficientes de exportación de nutrientes aportados por Johnes et al. (1996). Estos coeficientes han sido aplicados a los valores de superficie de utilización del suelo (actividad agrícola, uso forestal y uso pascícola), sin haber considerado otras actividades como por ejemplo la ganadería o los aportes procedentes de la presencia humana. Es importante hacer constar los elevados valores de aportación de ambos nutrientes que se han obtenido (Tabla 4). Aunque estos coeficientes no han sido obtenidos específicamente para la región Mediterránea, creemos que de

Tabla 4. Valores estimados mínimos y máximos de aportación de nutrientes (nitrógeno y fósforo total) en los humedales del Alto Guadalquivir, en función de los usos del territorio. Los cálculos se han realizado utilizando los coeficientes de exportación de nutrientes de Johnes et al. (1996). Minimum and maximum estimated values of the external nutrient loads (total nitrogen and total phosphorus) in the Alto Guadalquivir wetlands as a function of the land use. Calculations were based on the nutrient export coefficients described by Johnes et al. (1996).

\begin{tabular}{|c|c|c|c|c|c|c|c|c|c|c|c|}
\hline \multirow{3}{*}{$\begin{array}{c}\text { Humedal } \\
\text { Honda }\end{array}$} & \multicolumn{4}{|c|}{ Agricultura } & \multicolumn{4}{|c|}{ Pasto } & \multicolumn{3}{|c|}{ Uso Forestal } \\
\hline & \multicolumn{2}{|c|}{ Kg. N total } & \multicolumn{2}{|c|}{ Kg. P total } & \multicolumn{2}{|c|}{ Kg. N total } & \multicolumn{2}{|c|}{ Kg.P total } & \multirow{2}{*}{$\frac{K g . N \text { total }}{1.14}$} & \multicolumn{2}{|c|}{ Kg. P total } \\
\hline & 234.08 & 6061.00 & 56.848 & 482.372 & 1.687 & 6.44 & 0.07 & 0.49 & & 0.019 & 0.114 \\
\hline Chinche & 225.96 & 5850.75 & 54.876 & 465.639 & 4.579 & 17.48 & 0.19 & 1.33 & - & - & - \\
\hline Quinta & 197.96 & 5125.75 & 48.076 & 407.939 & - & - & - & - & 0.06 & 0.001 & 0.006 \\
\hline Rincón Muerto & 113.12 & 2929.00 & 27.472 & 233.108 & 7.953 & 30.36 & 0.33 & 2.31 & - & - & - \\
\hline Hituelo & 80.08 & 2073.50 & 19.448 & 165.022 & - & - & - & - & 0.24 & 0.004 & 0.024 \\
\hline Naranjeros & 285.04 & 7380.50 & 69.224 & 587.386 & 7.712 & 29.44 & 0.32 & 2.24 & 0.18 & 0.003 & 0.018 \\
\hline Las Ceras & 38.08 & 986.00 & 9.248 & 78.472 & - & - & - & - & 0.06 & 0.001 & 0.006 \\
\hline El Ranal & 546.28 & 14144.75 & 132.668 & 1125.727 & - & - & - & - & - & - & - \\
\hline Casillas & 63.56 & 1645.75 & 15.436 & 130.979 & - & - & - & - & - & - & - \\
\hline Las Navas & 189.28 & 4901.00 & 45.968 & 390.052 & - & - & - & - & - & - & - \\
\hline Villadonpardo & 153.72 & 3980.25 & 37.332 & 316.773 & - & - & - & - & 2.76 & 0.046 & 0.276 \\
\hline Garciez & 269.36 & 6974.50 & 65.416 & 555.074 & 4.097 & 15.64 & 0.17 & 1.19 & - & - & - \\
\hline Prados Moral & 42.84 & 1109.25 & 10.404 & 88.281 & - & - & - & - & 0.54 & 0.009 & 0.054 \\
\hline Brujuelo & 406.28 & 10519.75 & 98.668 & 837.227 & 7.953 & 30.36 & 0.33 & 2.31 & 4.50 & 0.075 & 0.45 \\
\hline Grande + Chica & 341.32 & 8837.75 & 82.892 & 703.363 & - & - & - & - & - & - & - \\
\hline Argamasilla & 115.92 & 3001.50 & 28.152 & 238.878 & - & - & - & - & 1.14 & 0.019 & 0.114 \\
\hline Santisteban & 49.56 & 1283.25 & 12.036 & 102.129 & 15.424 & 58.88 & 0.64 & 4.48 & - & - & - \\
\hline Perales & 36.68 & 949.75 & 8.908 & 75.587 & - & - & - & - & 0.66 & 0.011 & 0.066 \\
\hline Pedernoso & 35.84 & 928.00 & 8.704 & 73.856 & - & - & - & - & 1.26 & 0.021 & 0.126 \\
\hline Siles & - & - & - & - & 97.605 & 372.60 & 4.05 & 28.35 & 155.70 & 2.595 & 15.57 \\
\hline Orcera & - & - & - & - & - & - & - & - & 50.94 & 0.849 & 5.094 \\
\hline
\end{tabular}


modo cualitativo pueden constituir unas estimas de las tendencias de exportación de nutrientes hacia los ecosistemas acuáticos, observándose como éstas son siempre mayores para el caso de la agricultura frente a las otras actividades.

El efecto directo más inmediato asociado a este aporte de nutrientes es la eutrofización de los sistemas acuáticos, con repercusiones sobre la estructura, funcionamiento y diversidad de los ecosistemas. Esta contaminación difusa es característica de interacciones asimétricas como las que se establecen entre un ecosistema que se comporta como donante (cuenca de drenaje del humedal y donde se realizan los vertidos de productos agroquímicos) y un ecosistema receptor (sistema acuático per se). Por todo ello, el tamaño y las características de utilización de los usos del suelo en las cuencas de drenaje de las lagunas son determinantes sobre la calidad del agua y el estado actual de los humedales, poniéndose de manifiesto la importancia de un adecuado manejo de la unidad cuenca-humedal (incluyendo a las cuencas hidrológicas superficial y subterránea de alimentación) si se pretende conseguir una correcta gestión y conservación del patrimonio natural y la diversidad de los humedales.

En este sentido debemos indicar que resultados obtenidos en algunos de los ecosistemas aquí estudiados, como la laguna Honda (Castro, 2004) o las lagunas de la Quinta, Grande y Chica (Conde-Porcuna, com. per.), muestran un nivel de eutrofización que las sitúa en el rango de mesotróficas a hipertróficas. Igualmente, otras evidencias indirectas como la observación de un crecimiento excesivo de algas epifíticas sobre las praderas de macrófitos sumergidos (Becares et al., 2004); la desaparición de la vegetación sumergida (Van den Berg et al., 1999) y el aumento de la turbidez asociado a un incremento del fitoplancton o a la resuspensión de sedimentos ante la ausencia de vegetación acuática (Weisner et al., 1997), hechos que también han sido observadas en este conjunto de humedales, hacen denotar igualmente un elevado nivel de eutrofización de los mismos.

Por todo ello, si consideramos que para el caso de los humedales, la unidad funcional engloba la cuenca de drenaje, surge la necesidad de pa- sar de una gestión pasiva, como se ha ido practicando en la mayoría de los casos, a una gestión activa. Esta modalidad de gestión de los ecosistemas es más adecuada para los espacios naturales mediterráneos, en los que es necesario considerar tanto los valores naturales y culturales como la interacción entre ambos (García-Mora \& Montes, 2003). De este modo, los criterios ambientales de usos del territorio, bastante introducidos en relación con el urbanismo o la actividad industrial, deberían ganar peso progresivamente en relación con la actividad agrícola, de modo que estas intervenciones permitan mantener determinados cuadros de integridad ecológica.

La adopción de estas medidas de usos del territorio en relación con la actividad agrícola (Castle et al., 1999; Carreira \& García-Ruiz, 2005; Guerrero et al., 2005) o la aplicación de modelos eco-económicos para el análisis de las políticas agro-ambientales (Oglethorpe \& Sanderson, 1999) permitiría en un futuro mejorar la calidad de nuestros humedales.

\section{BIBLIOGRAFÍA}

ANTA, J. L. 2002. Parentesco y economía en una sociedad giennense En: La cultura del aceite en Andalucía. La tradición frente a la modernidad. J.L. Anta y J. Palacios (eds.): 161-170. Fundación Machado. Sevilla, España.

BECARES, E., A. CONTY, C, RODRÍGUEZ \& S. BLANCO. 2004. Funcionamiento de los lagos someros mediterráneos. Ecosistemas, 2004/2 (URL: http://www.aeet.org/ecosistemas/042/revision3.htm). BERNÁLDEZ, F. G. 1987. Las zonas encharcables españolas: el marco conceptual. En: Bases cientificas para la protección de los humedales en España. Real Academia de Ciencias Exactas, Físicas y Naturales (ed.): 9-30. Madrid, España.

BOUTIN, C. \& B. JOBIN. 1998. Intensity of agricultural practices and effects on adjacent habitats. Ecological Applications, 8: 544-557.

CARREIRA, J. \& R. GARCÍA-RUIZ. 2005. Principios ecológicos para la sostenibilidad de agroecosistemas y del olivar en particular: presentación de un marco conceptual general. En: La cultura del olivo: ecología, economía, sociedad. J. L. Anta, J. Palacios y F. Guerrero (eds.): 333-366. Universidad de Jaén, Jaén, España. 
CASTLE, K., C. FROST \& D. F. FLINT. 1999. The Loch Leven project - buffer strips in practice on a catchment scale. Aspects of Applied Biology, 54: 71-78.

CASTRO, M. C. 2004. Caracterización limnológica y variabilidad temporal de la comunidad planctónica en laguna Honda (Jaén). Tesis Doctoral. Universidad de Jaén. 164 pp.

CIRUJANO, S. 1995. Flora y vegetación de las lagunas y humedales de la provincia de Cuenca. Madrid. Consejo Superior de Investigaciones Científicas. 224 pp.

GARCÍA-MORA, M. R. \& C. MONTES. 2003. Vinculos en el paisaje mediterráneo: el papel de los espacios protegidos en el contexto territorial. Sevilla. Consejería de Medio Ambiente. Junta de Andalucía. 106 pp.

GIBSON, C. E., Y. WU \& D. PINKERTON. 1995. Substance budgets of an upland catchment: the significance of atmospheric phosphorus inputs. Freshwat. Biol., 33: 385-392.

GUERRERO, F., F. ORTEGA, G. PARRA, E. CANO, A. CANO, R. GARCÍA-RUIZ \& J. A. CARREIRA. 2002. Efectos ecológicos de la intensificación del cultivo del olivar en la comarca del Alto Guadalquivir: repercusiones sobre la diversidad. En: La cultura del aceite en Andalucía. La tradición frente a la modernidad. J. L. Anta y J. Palacios (eds.): 53-63. Fundación Machado. Sevilla, España.

GUERRERO, F., G. PARRA, F. JIMÉNEZ-GÓMEZ, M. C. CASTRO, R. JIMÉNEZ-MELERO, A. GALOTTI \& F. ORTEGA. 2005. Los ecosistemas acuáticos en el contexto de los agrosistemas: la comarca del Alto Guadalquivir. En: La cultura del olivo: ecología, economía, sociedad. J. L. Anta, J. Palacios y F. Guerrero (eds.): 381-402. Jaén. Universidad de Jaén.

INIMA. 1996. Lagos y humedales de España. Madrid. Ministerio de Obras Públicas, Transportes y Medio Ambiente. Dirección General de Obras Hidráulicas.

IRVINE, N., N. ALLOTT, P. MILLS \& G. FREE. 2000. The use of empirical relationships and nutrient export coefficients for predicting phosphorus concentrations in Irish lakes. Verh. Internat. Verein. Limnol., 27: 1127-1131.

ISTVÁNOVICS, V. \& L. SOMLYÓDY. 2001. Factors influencing lake recovery from eutrophicationthe case of basin 1 of lake Balaton. Wat. Res., 35: 729-735.
JOHNES, P., B. MOSS \& G. PHILLIPS. 1996. The determination of total nitrogen and total phosphorus concentrations in freshwaters from land use, stock headage and population data: testing of model for use in conservation and water quality management. Freshwat. Biol., 36: 451-473.

JUNTA DE ANDALUCÍA. 2003. Ortofotografía digital de Andalucía (color). Sevilla. Consejería de Medio Ambiente. Junta de Andalucía.

MADERO, A., F. ORTEGA \& F. GUERRERO. 2004. Lagunas y vías pecuarias en la provincia de Jaén: una nueva oportunidad para la conservación de los humedales. En: Biología de la Conservación. Reflexiones, propuestas y estudios desde el $S E$ ibérico. J. Peñas de Giles y L. Gutiérrez Cantero (eds.): 277-288. Instituto de Estudios Almerienses. Almería, España.

MÄLER, K. 2000. Development, ecological resources and their management: a study of complex dynamic systems. European Economic Review, 44: 645-665.

MATIAS, N. G. 2001. Estudo comparativo de duas albufeiras com influências sócio-económicas diferentes. Dissertação grau de Mestre. Universidade Técnica de Lisboa. 110 pp.

MOLINA, F., C. MONTES, E. GONZÁLEZCAPITEL \& J. C. RUBIO. C. 2002. El plan andaluz de humedales. Una estrategia para la conservación de los humedales en el siglo XXI. Medioambiente, 39: 14-19.

MORAGA, J. 2002. La reciente extensión del regadío en la provincia de Jaén. En: III Congreso Ibérico sobre la gestión y planificación del agua. La Directiva Marco del Agua, realidades y futuros. 13-17 Noviembre 2002. Universidad de Sevilla, Universidad Pablo de Olavide, Fundación nueva cultura del agua. Sevilla. España.

MORALES-BAQUERO, R., P. CARRILLO, I. RECHE \& P. SÁNCHEZ-CASTILLO. 1999. Nitrogen-phosphorus relationship in high mountain lakes: effects of the size of catchment basins. Can. J. Fish. Aquat. Sci., 56: 1809-1817.

MORALES-BAQUERO, R. \& J. M. CONDEPORCUNA. 2000. Effect of the catchment areas on the abundance of zooplankton in high mountain lakes of the Sierra Nevada (Spain). Verh. Internat. Verein. Limnol., 27: 1804-1808.

OGLENTHORPE, D. R. \& R. A. SANDERSON. 1999. An ecological-economic model for agrienvironmental policy analysis. Ecological Economics, 28: 245-266, 
ORS, J. 1999. Consideraciones sobre la ordenación del territorio en los ambientes fluctuantes. Caso de los humedales mediterráneos. Humedales Mediterráneos, 1: 99-106.

ORTEGA, F., G. PARRA. \& F. GUERRERO. 2003. Los humedales del Alto Guadalquivir: inventario, tipologías y estado de conservación. En: Ecología, manejo y conservación de los humedales. M. Paracuellos (ed.): 113-123. Instituto de Estudios Almerienses. Almería, España.

ORTEGA, F., G. PARRA \& F. GUERRERO. 2004. Las lagunas del Alto Guadalquivir: propuestas para su protección y conservación. En: Congreso de Restauración de ríos y humedales. J. Cachón y T. López-Piñeiro (eds.): 131-142. Cedex. Madrid, España.

ORTEGA, F., MADERO, A. \& GUERRERO, F. 2005. Las balsas de riego para el olivar: una alternativa a la destrucción de humedales. En: La cultura del olivo: ecología, economía, sociedad. J.L. Anta, J. Palacios y F. Guerrero (eds.): 435-448. Jaén. Universidad de Jaén.

PALACIOS, J. 2002. El olivar: la construcción de un texto híbrido. En: La cultura del aceite en Andalucía. La tradición frente a la modernidad.
J.L. Anta y J. Palacios (eds.): 25-49. Fundación Machado. Sevilla, España.

PASTOR, M., J. CASTRO \& M. D. HUMANES. 1996. Criterios para la elección de sistemas de cultivo en el olivar. Sevilla. Consejería de Agricultura y Pesca. Junta de Andalucía. 58 pp.

PORTA, J., M. LÓPEZ-ACEVEDO \& C. ROQUERO. 1994. Edafología para la agricultura y el medio ambiente. Madrid. Mundi-Prensa. 807 pp.

ULRICH, K. 1997. Effects of land use in the drainage area on phosphorus binding and mobility in the sediments of four drinking-water reservoirs. Hydrobiologia, 345: 21-38.

VAN DER BERG, M.S., M. SCHEFFER, E. VAN NESS \& H. COOPS. 1999. Dynamics and stability of Chara sp. and Potamogeton pectinatus in a shallow lake changing in eutrophication level. $\mathrm{Hy}$ drobiologia, 408/409: 335-342.

WEISNER, S. E. B., A. STRAND \& H. SANDSTEN. 1997. Mechanisms regulating abundance of submerged vegetation in shallow eutrophic lakes. Oecologia, 109: 592-599.

WILLIAMS, W. D. 1999. Conservation of wetlands in drylands: a key global issue. Aquatic Conservation: Marine and Freshwater Ecosystems, 9: 517-522. 